\title{
Dental Considerations in the Management of Meth Mouth: A Case Report
}

\author{
Narendra A Basutkar ${ }^{1}$, Asmaa Bokhari ${ }^{2}$, Othman Wali ${ }^{3}$
}

\begin{abstract}
Methamphetamine also popularly known as meth crystal, crystal, or ice is a highly addictive recreational drug that is a central nervous system stimulant. Even though the drug is banned, availability in the illegal black market because of its low cost and easy production technique makes it one of the most commonly used drugs by the addicts. Methamphetamine and amphetamine-type stimulants (ATS) like Captagon which contains fenethylline is chemically related to amphetamine, are powerful psychostimulant, produce an intense rush of pleasure, prolonged sense of euphoria, hyperactivity, increased awareness and hallucinations, convulsions and aggressiveness. Literature review shows patients with drug addiction have a typically dark, leathery distinctive type of rampant caries called methamphetamine-induced caries (MIC) along with xerostomia, gingival inflammation, and poor oral hygiene. Knowledge of methamphetamine drug abuse and its effect on dentition puts the dental practitioners in a unique position in identifying the patients with potential drug abuse. Dentists can play an important role in not only early detection but also can help patients in the cessation of the drug abuse.

Keywords: Drug abuse, Meth mouth, Methamphetamine.

International Journal of Prosthodontics and Restorative Dentistry (2020): 10.5005/jp-journals-10019-1272
\end{abstract}

\section{BACKGROUND}

Methamphetamine also popularly known as meth crystal, crystal, or ice is a highly addictive recreational drug that is a central nervous system stimulant. Even though the drug is banned, availability in the illegal black market because of its low cost and easy production technique makes it one of the most commonly used drugs by the addicts. ${ }^{1}$ Methamphetamine and amphetamine-type stimulants (ATS) like Captagon which contains fenethylline is chemically related to amphetamine, are powerful psychostimulant produces an intense rush of pleasure, prolonged sense of euphoria, hyperactivity, increased awareness and hallucinations, convulsions and aggressiveness. ${ }^{2}$

Literature review shows patients with drug addiction have a typically dark, leathery distinctive type of rampant caries called methamphetamine-induced caries (MIC) along with xerostomia, gingival inflammation, and poor oral hygiene. ${ }^{2,3}$ Knowledge of methamphetamine drug abuse and its effect on dentition puts the dental practitioners in a unique position in identifying the patients with potential drug abuse. Dentists can play an important role in not only early detection but also can help patients in the cessation of the drug abuse.

\section{Case Description}

A 26-year-old young male patient presented to comprehensive care clinics at Ibn Sina National College for Medical Studies, Jeddah, Saudi Arabia, for esthetic restorations for his badly broken down upper anterior teeth. His preoperative clinical presentation can be seen in pictures (Fig. 1).

The rampant nature of caries in the adult patient alarmed us. A detailed inquiry into history was made. Patient diet analysis was made. Upon further questioning, the patient admitted to Captagon addiction and had quit the same year back. Typical clinical presentation of rampant caries, poor oral hygiene, and a history of frequent intake of carbonated sugary drinks along with thorough patient history helped in establishing the diagnosis of Captagon drug abuse.

\footnotetext{
1,2Department of Prosthodontics, Ibn Sina National College for Medicals Studies, Jeddah, Kingdom of Saudi Arabia

${ }^{3}$ Department of Oral and Clinical Sciences, Ibn Sina National College for Medical Studies, Jeddah, Kingdom of Saudi Arabia
}

Corresponding Author: Narendra A Basutkar, Department of Prosthodontics, Ibn Sina National College for Medicals Studies, Jeddah, Kingdom of Saudi Arabia, Phone: +966557344673 , e-mail: narendra. basutkar@gmail.com

How to cite this article: Basutkar NA, Bokhari A, Wali O. Dental Considerations in the Management of Meth Mouth: A Case Report. Int J Prosthodont Restor Dent 2020;10(2):87-91.

Source of support: Nil

Conflict of interest: None

After the diagnosis was established, the patient was counseled and ill effects of the drug and on dental treatment were clearly mentioned to the patient. The fact that the patient had discontinued the abuse since 6 months ago, which was also confirmed by the family members, helped us to understand the patient is motivated enough to commit for the dental treatment. Patient motivation and commitment to good dental hygiene practices were the main focus of patient counseling.

A comprehensive dental treatment plan which included patient counseling was planned. To further reinforce the patient commitment and motivation to the dental treatment, digital smile designing was considered. Necessary frontal photographs were taken using a DSLR camera. DSD PowerPoint software and tools were used to complete the smile designing. Final results were shown to the patient and consent for the dental treatments was taken (Fig. 2).

After periapical X-rays (Fig. 3), the diagnosis was established and necessary extractions and non-surgical periodontal therapy and endodontic treatment were initiated. Plaque and bleeding scores were established preoperatively and were assessed during

() The Author(s). 2020 Open Access This article is distributed under the terms of the Creative Commons Attribution 4.0 International License (https://creativecommons. org/licenses/by-nc/4.0/), which permits unrestricted use, distribution, and non-commercial reproduction in any medium, provided you give appropriate credit to the original author(s) and the source, provide a link to the Creative Commons license, and indicate if changes were made. The Creative Commons Public Domain Dedication waiver (http://creativecommons.org/publicdomain/zero/1.0/) applies to the data made available in this article, unless otherwise stated. 


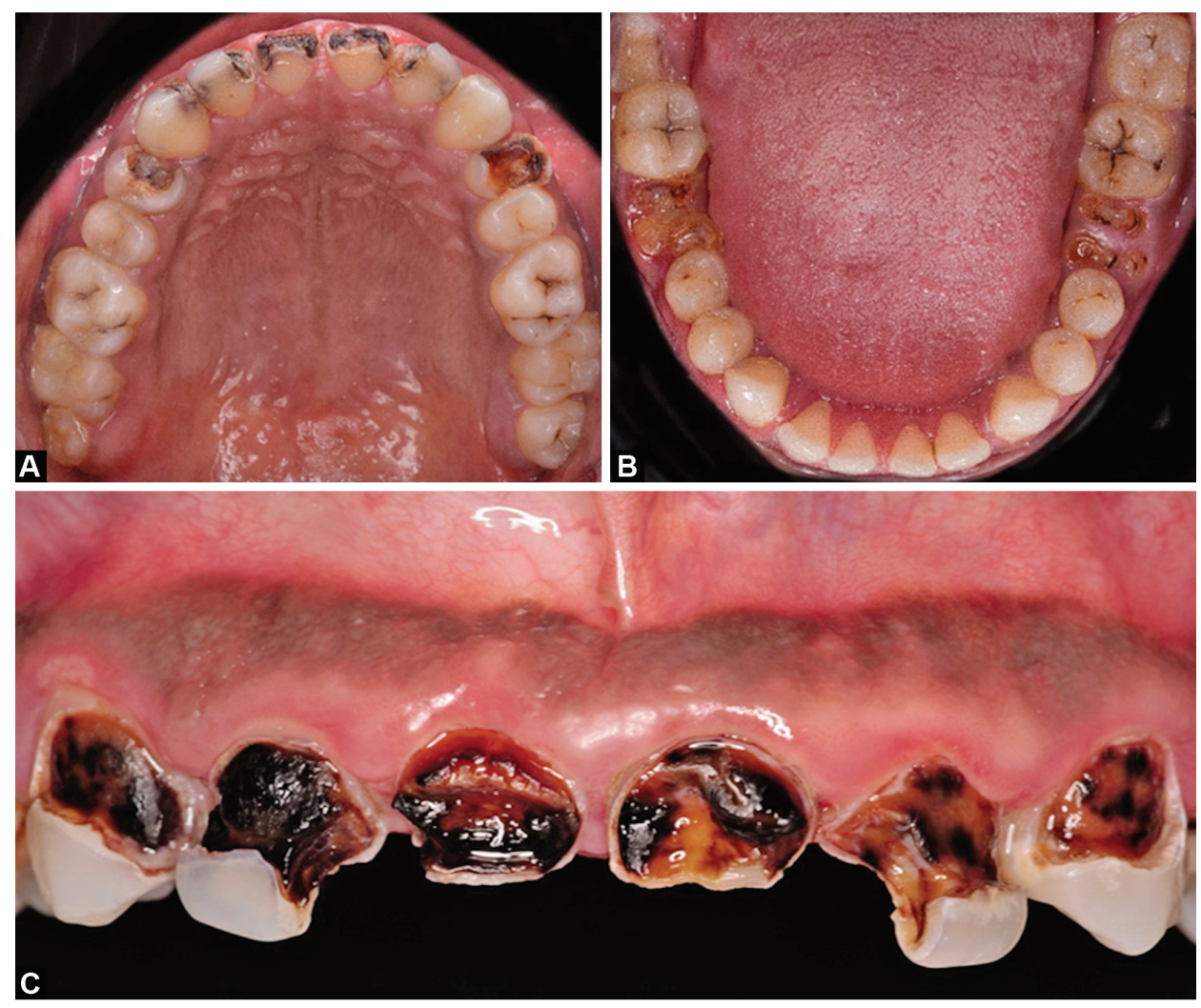

Figs $1 \mathrm{~A}$ to C: Preoperative photographs

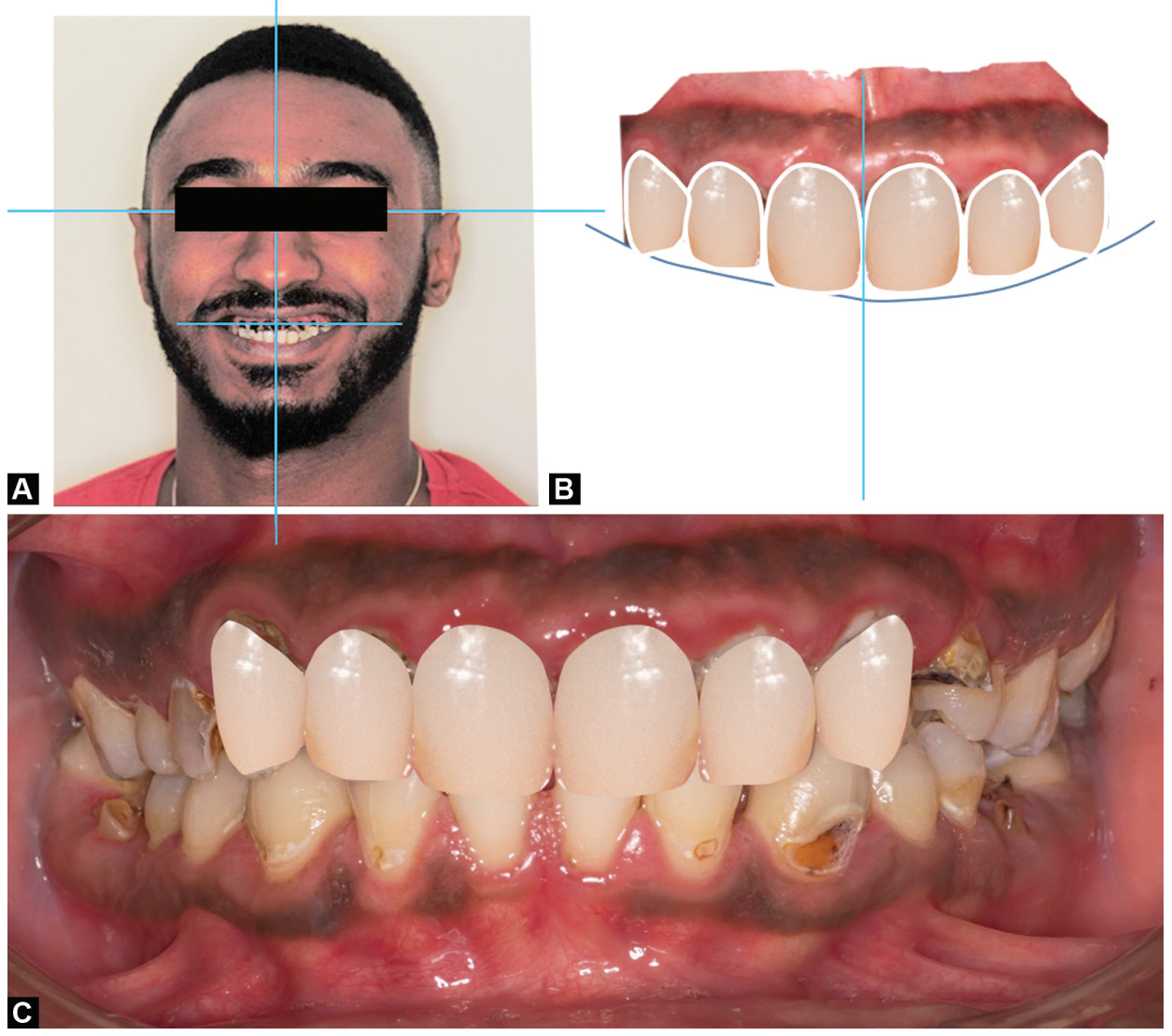

Figs $2 \mathrm{~A}$ to C: Digital smile designing 


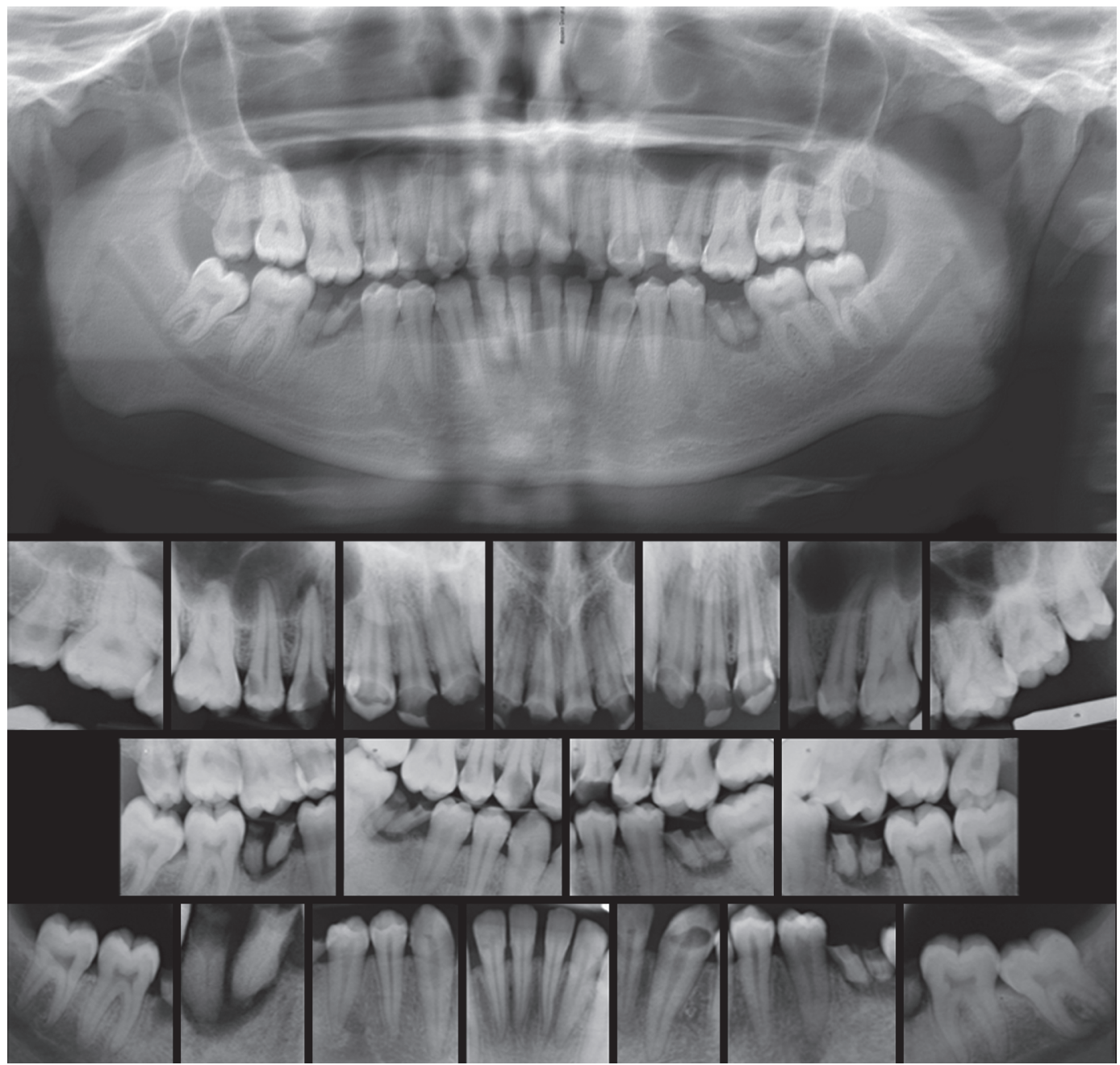

Fig. 3: Preoperative OPG and full mouth periapical radiographs

the phases of treatment regularly. This provided us with the much-needed information regarding oral hygiene practices of the patient during treatment phases. Reinforcement of oral hygiene instructions and patient motivation were carried out during the treatment phases.

After endodontic treatment of teeth numbers 11, 12, 21, and 22. Prefabricated fiber post (RelyX, 3M) was cemented and core (Luxacore Z, DMG America) was built. Teeth were prepared following all-ceramic guidelines to receive lithium disilicate (e.max, Ivoclar Vivadent) crowns. Final impressions were made using polyvinyl elastomeric impression material (3M, Pentamix) (Fig. 4). Crowns were cemented using resin cement (RelyX, 3M). Missing teeth in the mandibular arch were also replaced as part of a comprehensive treatment plan using metal-ceramic bridges. Final postoperative results can be seen in Figure 5.

\section{Discussion}

Drug abuse is a major concern all over the world. Like many other wealthy countries, the Kingdom of Saudi Arabia is frequently a target of drug smugglers. In its 2014 annual report, the international narcotics control board, clearly indicates that this is an increasingly serious problem in Saudi Arabia and other Gulf countries. ${ }^{1}$ Saudi Arabia has zero tolerance for drug abuse and drug handling in the country. The laws against drug sale and addiction are very stringent due to health and religious beliefs. Even after such strict laws, according to the published news reports, the drug abuse of methamphetamine and ATS drugs like Captagon locally known in Arabic as "Abu-Hilalain" is high. Because of the strict laws and punishments, many drug addicts unwilling to admit and many cases go unreported.

Usually the addicts seek dentist consultation to address their dental issues. This puts the onus on the dentist to identify the potential drug abuse and provide first-line counseling to the patient. Dentists can use this patient necessity to motivate and counsel patients against drug abuse. Review of literature shows that researchers have associated the rampant dental caries found in people who habitually used meth to a combination of factors, including xerostomia, frequent sipping of sugary soft drinks to relieve dry mouth symptoms, the corrosive effects of meth contaminants, and poor oral hygiene. ${ }^{2}$ Shaner et al., have reported that associated xerostomia in meth users could be attributed to a vasoconstriction of the salivary gland vasculature and a resultant reduction in salivary flow. ${ }^{3,4}$

In xerostomia, buffering action of saliva is absent or diminished, causing difficulty in speaking, swallowing, and eating. This results in a person consuming more liquids to alleviate the dry mouth symptoms. ${ }^{5}$ Morio et al., in their pilot study, comparing diet, oral hygiene, and caries status of adult methamphetamine users 

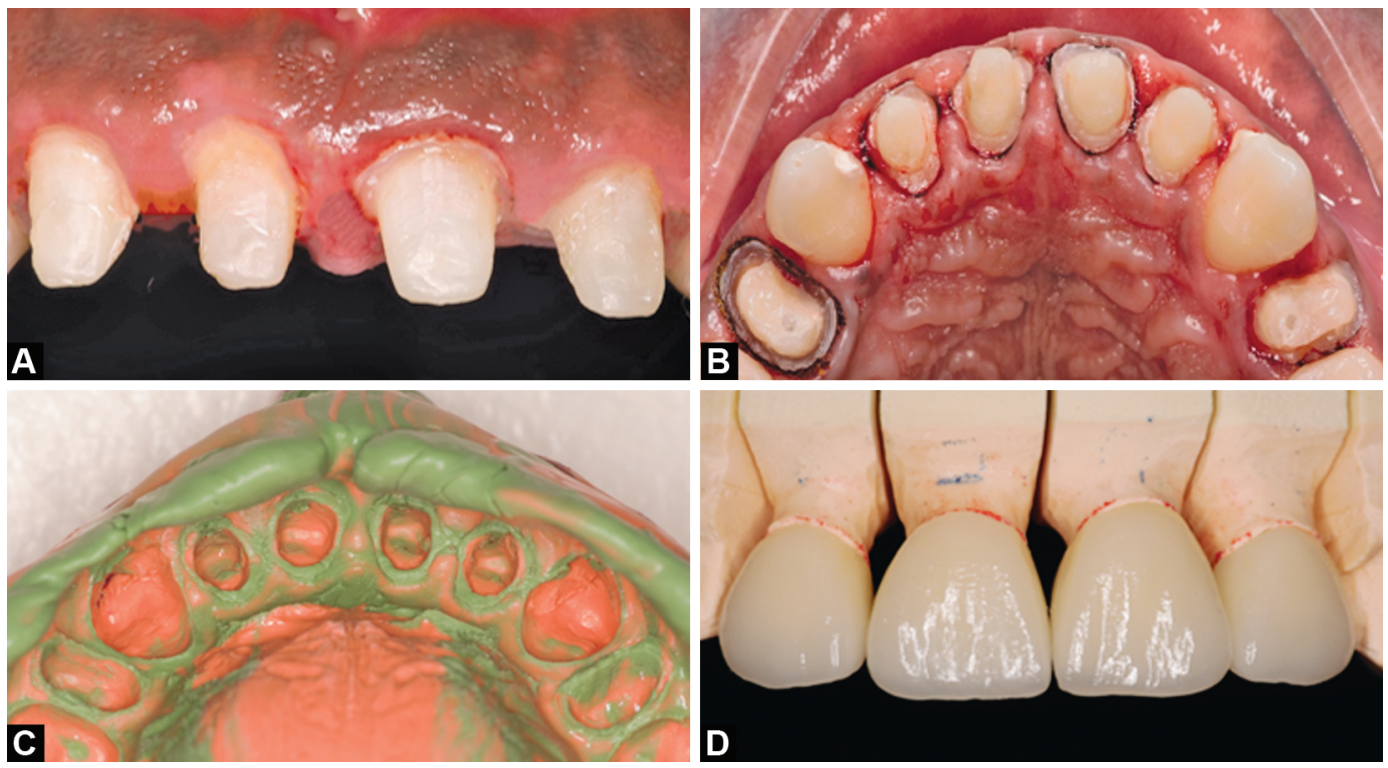

Figs $4 A$ to $D$ : Teeth preparations and impression
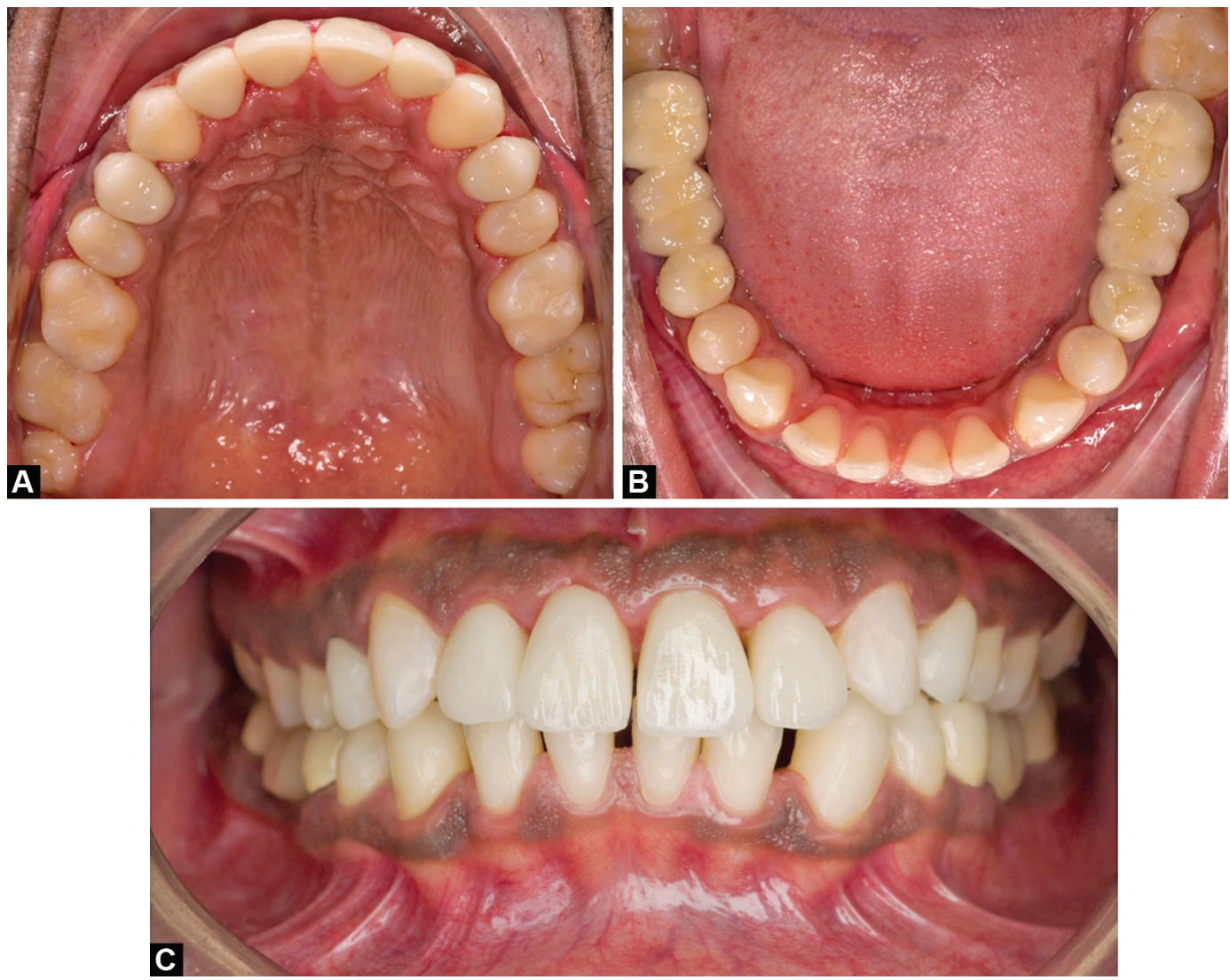

Figs 5A to C: Postoperative photographs

and non-users: Reported in 2008 found that, $94 \%$ of people who used meth stated that they frequently consumed sugary soda. ${ }^{2}$ The reduced protective buffering capacity of saliva leads to the widespread development of caries in meth abusers. Typically caries is seen on the facial and cervical surfaces of both maxillary and mandibular teeth. Usually caries progressively involve the entire clinical crown. This caries experience is often referred to as MIC. Usually caries progresses slowly in meth abusers because of the cyclical nature of oral hygiene practices during weeks of abuse and abstinence.

To explain the relationship between smoking meth and dental disease, some investigators have suggested that smoking meth creates an acidic environment in the mouth and contributes to dental caries. ${ }^{6}$ Others have claimed that the harsh chemicals used in the preparation of meth end up corroding the teeth, especially when a person smokes meth. ${ }^{4}$ On contrary to this popular 
perception, Clague et al., reported smoking meth does not produce greater rates of caries and xerostomia than snorting or injecting meth. ${ }^{7}$ The general consensus is observed in the literature between investigators regarding oral health behaviors in people who use meth like frequent intake of sugary drinks, poor diet infrequent toothbrushing, and poor oral hygiene. Investigators have suggested that these oral health behaviors play a key role in dental caries observed in people who use meth. ${ }^{2,4,6,8}$

A comprehensive approach protocol should be followed while managing the patient with meth and other ATS drug addiction. No definitive treatment should be initiated unless the drug abuse is established and proper counseling is initiated. Patient motivation to seek dental treatment for cosmetic change should be used as an advantage in patient counseling. III effects of the meth abuse and its effect on providing proper dental care should be informed to the patient. Non-surgical periodontal therapy involving patient education for correct methods of oral hygiene practices, scaling, and fluoridated mouth rinses should be considered first. Caries control and temporary restorations using fluoride-releasing glass ionomer cement should be initiated. Final composite restorations should be delayed until the patient shows promising progress with de-addiction and good oral hygiene practice. Definitive prosthetic treatments like crown and bridgework and removable prosthesis should be only considered after drug abuse is completely stopped.

\section{Conclusion}

Dental management of patients who use methamphetamine, ATS drugs can be challenging. Being aware of the ill effects of methamphetamine drug abuse on dentition puts dentists in a unique position to be able to detect addiction early and propose intervention. Dentists can use patient motivation to receive dental treatment for cosmetic purposes as a great motivational tool to educate patients with ill effects of methamphetamine and ATS drug abuse. Dentists need to be aware of the clinical presentation and medical risks presented by these patients.

\section{References}

1. International Narcotics Control Board. Report of the International Narcotics Control Board for 2014. 2014. Available from: https://www. unodc.org/documents/southeastasiaandpacific//Publications/2015/ incb/INCB_Annual_Report_2014_EN.pdf.

2. Morio KA, Teresa MA, Qian F, et al. Comparing diet, oral hygiene and caries status of adult methamphetamine users and nonusers: a pilot study. J Am Dent Assoc 2008;139(2):171-176. DOI: 10.14219/jada. archive.2008.0133.

3. Shaner JW. Caries associated with methamphetamine abuse. J Mich Dent Assoc 2002;84(9):42-47.

4. Klasser GD, Epstein J. Methamphetamine and its impact on dental care. J Can Dent Assoc 2005;71(10):759-762.

5. Greenspan D. Xerostomia: diagnosis and management. Oncology (Williston Park) 1996;10(3 suppl):7-11.

6. De-Carolis C, Boyd GA, Mancinelli L, et al. Methamphetamine abuse and "meth mouth" in Europe. Med Oral Patol Oral Cir Bucal 2015;20(2):e205-e210. DOI: 10.4317/medoral.20204.

7. Clague J, Belin TR, Shetty V. Mechanism's underlying methamphetamine-related dental disease. J Am Dent Assoc 2017;148(6):377-386. DOI: 10.1016/j.adaj.2017.02.054

8. Shaner JW, Kimmes NS, Saini T, et al. "Meth mouth": rampant caries in methamphetamine abusers. AIDS Patient Care and STDS 2006;20(3):146-150. DOI: 10.1089/apc.2006.20.146. 